\title{
Avaliação dos fatores de risco para fatura osteoporótica em mulheres pós menopausa
}

\author{
Evaluation of risk factors for osteoporotic bill in postmenopausal women \\ Evaluación de factores de riesgo de factura osteoporótica en mujeres posmenopáusicas
}

Recebido: 10/05/2021 | Revisado: 16/05/2021 | Aceito: 17/05/2021 | Publicado: 06/06/2021

\author{
Breno William Santana Alves \\ ORCID: https://orcid.org/0000-0001-9869-8731 \\ Universidade Tiradentes, Brasil \\ E-mail: breno.william@ hotmail.com \\ Francisco de Assis Pereira \\ ORCID: https://orcid.org/0000-0002-8464-6188 \\ Universidade Federal de Sergipe, Brasil \\ E-mail: frasp@terra.com.br \\ Ronald Bispo Barreto da Silva \\ ORCID: https://orcid.org/0000-0002-8500-1904 \\ Universidade Tiradentes, Brasil \\ E-mail: dr.ronaldbarreto@gmail.com \\ José Seabra Alves Neto \\ ORCID: https://orcid.org/0000-0001-6289-2911 \\ Universidade Tiradentes, Brasil \\ E-mail: seabraneto96@gmail.com \\ Amanda Raquel Costa Cruz \\ ORCID: https://orcid.org/0000-0003-0192-745X \\ Universidade Tiradentes, Brasil \\ E-mail: amandarccruz@gmail.com
}

\begin{abstract}
Resumo
Objetivo: avaliar os fatores de risco para osteoporose e fratura por fragilidade em mulheres idosas. Métodos: Estudo descritivo, observacional e transversal com amostra de conveniência realizado em um serviço ambulatorial, privado, vinculado à Universidade Tiradentes, na cidade de Aracaju-SE. Resultados: A casuística do estudo foi composta por 102 mulheres pós menopausa. Os três fatores de risco para osteoporose que apresentaram maior frequência em nossa amostra foram sedentarismo (70,6\%), uso de corticoide $(17,6 \%)$, menopausa precoce $(15,3 \%)$, todas as participantes possuíam pelo menos três fatores de risco. Todas as participantes do estudo têm indicação de densitometria óssea, porém $68(66,7 \%)$ referiram ter sido submetidas ao exame e destas, $32(31,4 \%)$ referiram ter o diagnóstico de osteoporose densitométrica. Em relação à queda, 43 (42,2\%) participantes relataram já ter apresentado pelo menos um episódio no último ano. Conclusões: Nossos resultados demonstram que a população feminina e idosa, embora apresente maiores risco para osteoporose e fratura por fragilidade, ainda é negligenciada em nosso meio, demonstrando que há necessidade de programas de promoção de saúde e na prática médica rotineira para detecção dos fatores de risco para osteoporose e bem com de fraturas nos grupos de indivíduos mais propensos a apresentarem estas condições clínicas.
\end{abstract}

Palavras-chave: Osteoporose; Fratura; Idoso.

\begin{abstract}
Objective: to evaluate the risk factors for osteoporosis and fragility fracture in elderly women. Methods: Descriptive, observational and cross-sectional study with a convenience sample carried out in an outpatient, private service, linked to the Tiradentes University, in the city of Aracaju-SE. Results: The study sample consisted of 102 post-menopausal women. The three risk factors for osteoporosis that were more frequent in our sample were sedentary lifestyle (70.6\%), use of corticosteroids (17.6\%), early menopause (15.3\%), all participants had at least three factors risk. All study participants have an indication for bone densitometry, however 68 (66.7\%) reported having undergone the examination and of these, 32 (31.4\%) reported having a diagnosis of densitometric osteoporosis. Regarding the fall, $43(42.2 \%)$ participants reported having had at least one episode in the last year. Conclusions: Our results demonstrate that the female and elderly population, although presenting greater risk for osteoporosis and fragility fracture, is still neglected in our country, demonstrating that there is a need for health promotion programs and routine medical practice to detect the factors of risk for osteoporosis and fractures in the groups of individuals most likely to have these clinical conditions.
\end{abstract}

Keywords: Osteoporosis; Fracture; Elderly. 


\begin{abstract}
Resumen
Objetivo: evaluar los factores de riesgo de osteoporosis y fractura por fragilidad en mujeres ancianas. Métodos: Estudio descriptivo, observacional y transversal con muestra de conveniencia realizado en un servicio ambulatorio, privado, vinculado a la Universidad Tiradentes, en la ciudad de Aracaju-SE. Resultados: La muestra del estudio consistió en 102 mujeres posmenopáusicas. Los tres factores de riesgo de osteoporosis que fueron más frecuentes en nuestra muestra fueron el sedentarismo $(70,6 \%)$, el uso de corticosteroides $(17,6 \%)$, la menopausia precoz $(15,3 \%)$, todas las participantes tenían al menos tres factores de riesgo. Todos los participantes del estudio tienen indicación de densitometría ósea, sin embargo 68 (66,7\%) informaron haberse sometido al examen y de estos, 32 (31,4\%) informaron tener un diagnóstico de osteoporosis densitométrica. En cuanto a la caída, 43 (42,2\%) participantes informaron haber tenido al menos un episodio en el último año. Conclusiones: Nuestros resultados demuestran que la población femenina y anciana, aunque presenta mayor riesgo de osteoporosis y fractura por fragilidad, sigue siendo desatendida en nuestro país, demostrando que existe la necesidad de programas de promoción de la salud y práctica médica rutinaria para detectar los factores de riesgo de osteoporosis y fracturas en los grupos de personas con mayor probabilidad de tener estas condiciones clínicas.
\end{abstract}

Palabras clave: Osteoporosis; Fractura; Anciano.

\title{
1. Introdução
}

A osteoporose é considerada uma doença decorrente de múltiplos mecanismos patogenéticos que convergem para perda de massa óssea e a deterioração da microarquitetura da estrutura esquelética, com consequente aumento da propensão para fraturas de fragilidade (NIH, 2001; Kanis et al., 2019). A osteoporose e as fraturas decorrentes dela são consideradas como grandes problemas de saúde pública devido a sua frequência e gravidade, além dos altos custos pessoais, sociais e morbimortalidade na população, ocasionando redução da qualidade e expectativa de vida (Kanis et al., 2019; Radominski et al., 2017).

Estima-se que a osteoporose afete 200 milhões de mulheres no mundo (Radominski et al., 2017). A incidência de fraturas por fragilidade é mais de 8,9 milhões por ano e $28 \%$ das mulheres com osteoporose terão fraturas de quadril e $25 \%$ terão fraturas vertebrais (Barrionuevo et al., 2019). Na América Latina, os estudos mostram prevalência de fratura de quadril de 4 a 36,2 por 10 mil pessoas (Osteoporosis, 1995). No Brasil, cerca de 10 milhões de habitantes têm osteoporose e alta prevalência de fraturas por fragilidade nos diferentes sítios ósseos (coluna vertebral, quadril, antebraço e úmero proximal), variando de 11 a 23,8\% e está associada à ocorrência de quedas (Moraes et al., 2014; Pinheiro et al.,2010).

No estudo SAPOS, envolvendo 4.332 mulheres acima de 40 anos evidenciou que a prevalência de osteoporose pósmenopausa foi de $33 \%$ e fratura por fragilidade em $11,5 \%$ dessa população. As fraturas vertebrais e de quadril foram referidas por $6 \%$ e $8 \%$ das mulheres, respectivamente; neste estudo $15 \%$ da casuística relatou história familiar de fratura de quadril em parentes de primeiro grau (Pinheiro et al., 2010).

O tratamento da osteoporose e de suas complicações são elevados. No Brasil nos anos de 2008-2010 chegaram perto dos 300 milhões de reais (Moraes et al., 2014). No estado de Sergipe, o gasto relacionado ao tratamento para fraturas de quadril no período de 2000-2014 foi 8.960.826,00 reais (Pereira et al., 2018). Diante da alta prevalência e morbimortalidade decorrente da osteoporose e das fraturas por fragilidade, a avaliação dos fatores de risco para osteoporose na prática clínica é de fundamental importância. Existem fatores que se associam com maior valor preditivo para risco de osteoporose e de fratura, como: idade, baixo peso, fratura osteoporótica prévia, uso de glicocorticoide, uso de alguns anticonvulsivantes, sedentarismo, hiperparatireoidismo primário, hipogonadismo (Maclean et al., 2007; Frazão \& Naveira ,2006).

O estudo NORA, mostrou que os principais fatores de risco para fraturas por fragilidade foram baixa da massa óssea, idade avançada, história de fratura prévia, história materna de fratura, ser da raça branca, usar glicocorticoide, fumar ou ter fumado (Siris et al., 2001) No estudo BRAZOS os principais fatores de risco para fraturas nas mulheres foram idade avançada, história familiar de fratura de fêmur, menopausa precoce, sedentarismo, baixa qualidade de vida, maior ingestão de fósforo, diabetes mellitus, uso de benzodiazepínicos e quedas recorrentes (Pinheiro et al., 2010).

O objetivo deste estudo foi avaliar os fatores de risco para osteoporose e fratura por fragilidade em mulheres idosas. 


\section{Metodologia}

Trata-se de um estudo transversal, descritivo, amostra de conveniência e com abordagem quantitativa (Pereira, et al.,2018) realizado em um serviço ambulatorial, privado, vinculado à Universidade Tiradentes, na cidade de Aracaju-SE. Este estudo foi aprovado pelo Comitê de Ética e Pesquisa da Universidade Tiradentes (CAAE No 73275417.8.0000.5371), parecer número: 2.391.824.

As pacientes foram selecionadas de acordo com os critérios de inclusão: idade igual ou superior a 65 anos de ambos os sexos, com condições clínicas e cognitivas que permitissem responder ao questionário e que concordaram em participar da pesquisa.

O instrumento de coleta de dados utilizado foi um questionário estruturado e desenvolvido especialmente para esse estudo que aborda dados demográficos [sexo, raça, peso, altura, Índice de Massa Corporal (IMC)] fatores de risco para osteoporose [menopausa precoce, sedentarismo, uso de corticoide, uso de anticonvulsivante, fratura prévia, antecedente familiar para osteoporose, tabagismo e etilismo, fratura de quadril em pais, síndrome de má absorção, Doença Pulmonar Obstrutiva Crônica (DPOC)], evento de queda.

Foram considerados tabagistas os pacientes que fizeram uso de, pelo menos, um cigarro diário, por período não inferior a um mês, ou aquele que cessou o hábito de fumar com menos de 12 meses, de acordo com as normas da Sociedade Brasileira de Pneumologia e Tisiologia. E como etilistas, foram considerados os indivíduos que ingeriam bebida alcoólica mais que duas vezes por semana, independentemente do tipo e da quantidade.

Para a análise estatística dos dados obtidos, utilizou-se o programa GraphPad Prism, versão 7.0 para Windows, 2016 (San Diego, CA, USA). Os resultados foram apresentados como média e desvio-padrão.

\section{Resultados e Discussão}

A casuística do estudo foi composta por 102 mulheres pós menopausa. Em relação à raça, 54 (55,1\%) eram não brancas. A média de peso, altura e IMC foram $66,0 \pm 8,0 \mathrm{~kg}, 1,53 \pm 0,2 \mathrm{~m}$, e 27,2 $\pm 3,8 \mathrm{~kg} / \mathrm{m} 2$ respectivamente.

Os fatores de risco para osteoporose que apresentaram maior frequência em nossa amostra foram sedentarismo, uso de corticoide, artrite reumatoide, menopausa precoce, tabagismo, etilismo, fratura de quadril em pais, uso de anticonvulsivante, DPOC e síndrome de má absorção como apresentados na Tabela 1. Todas as participantes possuíam pelo menos três fatores de risco 
Tabela 1. Distribuição dos fatores de risco para osteoporose nas participantes do estudo. ( $\mathrm{N}=102)$.

\begin{tabular}{l|l|l}
\hline $\begin{array}{l}\text { FATORES DE RISCO PARA } \\
\text { OSTEOPOROSE }\end{array}$ & $\begin{array}{l}\text { TODAS AS } \\
\text { PARTICIPANTES } \\
\text { N= 129 } \\
\text { n }(\%) \\
72(70,6)\end{array}$ & $\begin{array}{l}\text { PARTICIPANTES } \\
\text { COM OSTEOPOROSE } \\
\text { N = 32 } \\
\mathbf{n}(\%) \\
25(78,1)\end{array}$ \\
Sedentarismo & $18(17,6)$ & $12(37,5)$ \\
Uso de corticoide & $15(15,3)$ & $7(21,9)$ \\
Menopausa Precoce $(<45$ anos $)$ & $8(7,9)$ & $6(18,8)$ \\
Fratura de quadril em pais & $8(7,9)$ & $6(18,8)$ \\
Artrite Reumatoide & $7(6,9)$ & $5(15,6)$ \\
Tabagismo & $5(4,9)$ & $5(15,6)$ \\
DPOC & $4(3,9)$ & $2(6,3)$ \\
Etilismo & $2(1,9)$ & $1(3,1)$ \\
Síndrome de má absorção & $2(1,4)$ & $1(3,1)$ \\
\hline Uso de anticonvulsivante & & \\
\hline
\end{tabular}

n: número, \%: porcentagem. Fonte: Respostas coletadas por meio de questionário para a pesquisa.

Das 102 participantes do estudo, 68 (66,7\%) referiram ter sido submetidas ao exame de densitometria óssea e destas, $32(31,4 \%)$ referiram ter o diagnóstico de osteoporose densitométrica. Nenhuma paciente avaliada apresentava diagnóstico de fratura osteoporótica de fêmur ou punho, porém todas referiram redução da altura maior do que 4 centímetros, em relação à altura, quando jovens.

Em relação à queda, 43 (42,2\%) participantes relataram já ter apresentado pelo menos um episódio no último ano. A distribuição de quedas por faixa etária está apresentada na Tabela 2. Este evento esteve presente em 19 (59,4\%) das participantes com diagnóstico de osteoporose densitométrica.

Em relação ao tratamento para osteoporose, das 32 participantes, 15 (46,9\%) estavam em tratamento com bisfosfonato há pelo menos três anos, porém com períodos de interrupção, seis $(18,8 \%)$ participantes relataram uso regular da medicação no período dos últimos três anos, nove $(28,1 \%)$ relataram já ter realizado tratamento para osteoporose, porém interrompeu por conta própria ou por intolerância à medicação e duas $(6,2 \%)$ pacientes referiram nunca ter iniciado o tratamento.

Tabela 2. Avaliação de episódio quedas em relação a faixa etária $(\mathrm{N}=102)$.

\begin{tabular}{lll}
\hline FAIXA ETÁRIA (a) & $\mathbf{n}$ & EPISÓDIO DE QUEDA (\%) \\
$\mathbf{6 5}-\mathbf{6 9}$ a & 53 & $22(41,5)$ \\
$\mathbf{7 0}-\mathbf{7 9}$ a & 36 & $15(41,7)$ \\
$\mathbf{8 0}-\mathbf{9 0}$ a & 13 & $6(46,2)$ \\
TOTAL & $\mathbf{1 0 2}$ & $\mathbf{4 3 ( 4 2 , 2 \% )}$ \\
\hline
\end{tabular}

a: anos; n: número. Fonte: Respostas coletadas por meio de questionário para a pesquisa. 
A osteoporose é um problema de saúde pública mundial cuja importância e impacto vem crescendo (Turner et al., 2018). Nas últimas décadas houve importante avanço na compreensão da fisiopatolgia da osteoprose e consequentemente novos tratamentos estão disponiveis, entretanto há necessidade de que seu diagnóstico seja adequadamente realizado para redução das suas complicações e do seu impacto social e econômico (Sernbo \& Johnell, 2003). Desta forma a aplicação de programas de triagem comunitária são necessárias para que a osteoporose não seja uma doença subdiagnosticada e subtratada em diferentes partes do mundo (Vestergaard et al., 2005; Santana Júnior et al., 2017; Alves Neto et al.,2018).

O objetivo deste estudo é identificar os fatores de risco para osteoporose e fratura por fragilidade em idosas na cidade de Aracaju - SE, uma vez que em estudos anteriores realizados no estado de Sergipe detectaram que a osteoporose é negligenciada Santana Júnior et al., 2017; Alves Neto et al.,2018). No presente estudo realizado com 102 mulheres idosas, observamos que $68(66,7 \%)$ foram submetidas à densitometria óssea, quando pela recomendação da OMS, todas deveriam ter realizado o exame para rastreamento da osteoporose.

Este achado corrobora o fato que o rastreamento populacional com exame de densitometria óssea não é factível, especialmente em países pobres ou em desenvolvimento, decorrente dos custos elevados (Kanis, 2007; Szejnfeld et al., 2007). Adicionalmente, o acesso à densitometria óssea pelo SUS ainda é precário, uma vez que apenas em 9,6\% dos municípios brasileiros possuem o equipamento (Arruda et al., 2013; Pereira 2017). Desta forma, a avaliação clínica dos fatores de risco para osteoporose e fratura por fragilidade é útil quando a densitometria óssea não for acessível.

Em nossa casuística, avaliamos somente mulheres com idade superior a 65 anos e os principais fatores de risco para osteoporose e fratura por fragilidade foram sedentarismo, uso de corticoide e menopausa precoce. Estes critérios de mulher e idosa podem ter contribuído para alta prevalência dos fatores de risco para osteoporose e fratura por fragilidade, fato este, bem descrito na literatura (Rubin et al.,, 2018; Ralston \& Fraser,2015). No estudo BRAZOS, sedentarismo, tabagismo atual, pior qualidade de vida e diabetes mellitus foram os fatores de risco mais relevantes para fratura por fragilidade em homens brasileiros. Nas mulheres, os mais importantes foram idade avançada, menopausa precoce, sedentarismo, pior qualidade de vida, maior consumo de fósforo, diabete melito, quedas, uso crônico de benzodiazepínicos e história familiar de fratura de fêmur após os 50 anos em parentes de primeiro grau. Esses fatores de risco refletem o envolvimento de hereditariedade, hábitos de vida, qualidade de vida, quedas e o próprio envelhecimento como determinantes na deterioração da qualidade óssea e maior risco de fratura (Pinheiro et al., 2010)

Diversos estudos brasileiros com amostragem não representativa da população brasileira, encontraram variados fatores de risco associados à osteoporose, como menopausa precoce, idade avançada, baixo peso e baixa estatura, menor IMC, tabagismo, ausência de terapia hormonal após a menopausa, baixa exposição solar, consumo de bebidas alcoólicas, ingestão inadequada de cálcio, sedentarismo, história familiar de osteoporose (Pinheiro et al., 2010; Pinheiro et al., 2006; Lanzillotti et al., 2003).

Com o envelhecimento populacional, as quedas têm apresentado relevante impacto na saúde pública decorrente de suas comorbidades: trauma cranioencefálico, hospitalização, institucionalização, fraturas e óbito (Pinheiro \& Ragi, 2010). Em idosos, a prevalência de quedas recorrentes no último ano pode variar de 10 a $40 \%$ (Fabrício et al., 2004). Quando avaliamos o evento queda, nossos dados demonstram ser um evento comum na população estudada, com prevalência de $42,2 \%$. A avaliação de queda e de risco de quedas na população idosa deve ser um aspecto abordado na prática médica rotineira, pois a associação de quedas e fraturas é significante, sendo a queda o principal fator de risco para fratura (Rodrigues et al., 2005).

Um dado que chama atenção em nosso estudo é que somente é que somente 18,8\% das pacientes com osteoporose estavam sendo adequadamente tratada para osteoporose. A negligência em relação à osteoporose é um grande problema de saúde pública em muitos países, incluindo o Brasil (Takkouche et al., 2007). O impacto da osteoporose e da fratura sobre redução de qualidade de vida, incapacidade, morbidade, mortalidade e alto custo econômico e que o seu adequado diagnóstico 
e tratamento são capazes de reduzir o risco de fratura vertebral e não vertebral em torno de $65 \%$ e $40 \%$ respectivamente são bem estabelecidos (Fortes et al., 2008).

Nosso estudo apresenta limitações, entre elas, o número de participantes da pesquisa, a falta da realização da densitometria óssea para correlacionar o diagnóstico densitométrico com os fatores de risco para osteoporose. Estudos futuros com desenho longitudinal, realização de densitometria óssea são necessários para melhor avaliação do risco de fratura por fragilidade. Entretanto, nossos resultados indicam que há necessidade de uma melhor avaliação médica com o objetivo de rastrear indivíduos com maior risco para osteoporose e fratura por fragilidade em nosso meio e indicar com melhor precisão a realização da densitometria óssea, oferecer orientações quanto a prevenção de quedas para diminuir o risco de fraturas e suas complicações.

\section{Conclusão}

Nossos resultados demonstram que a população feminina e idosa, embora apresente maiores risco para osteoporose e fratura por fragilidade, ainda é negligenciada em nosso meio, demonstrando que há necessidade de programas de promoção de saúde e na prática médica rotineira para detecção dos fatores de risco para osteoporose e bem com de fraturas nos grupos de indivíduos mais propensos a apresentarem estas condições clínicas para auxiliar na tomada de decisão de intervenção individualizada.

\section{Referências}

Advisors and National Societies of the International Osteoporosis Foundation (IOF). European guidance for the diagnosis and management of osteoporosis in postmenopausal women. Osteoporosis International, 30(1):3-44

Alves Neto, J. S., Cruz, A. R. C., Pereira, F. A. \& Pereira, A. F. P. B. (2018). Avaliação dos fatores de risco para osteoporose em idosos atendidos em unidade de saúde da universidade Tireadentes. Poster apresentado no $8^{\circ}$ Congresso Brasileiro de Densitometria, Osteoporose e osteometabolismo da Associação Brasileira de Avaliação Óssea e Osteometabolismo.

Arruda, M. F., Pereira, L. G. A., Fávero, T. M., Cassettari, L. L. \& de Souza Junior, A. P. (2013). Perfil do Uso do Programa Avaliacional Sapori em Paralelo à Necessidade da Densitometria Mineral Óssea em Amostra de Mulheres Pós-Menopáusicas da Cidade de Catanduva-SP. Saúde e Pesquisa, 6(2).

Barrionuevo, P., Kapoor, E., Asi, N., Alahdab, F., Mohammed, K., Benkhadra, K., Almasri, J., Farah, W., Sarigianni, M., Muthusamy, K., Al Nofal, A., Haydour, Q., Wang, Z. \& Murad, M.H. (2019). Efficacy of Pharmacological Therapies for the Prevention of Fractures in Postmenopausal Women: A Network Meta-Analysis. The jornal of clinical endocrinology and metabolismo, 1; 104(5):1623-1630.

Effectiveness of Screening in the Community to Reduce Osteoporotic Fractures in Older Women in the UK: Economic Evaluation of the SCOOP Study. Journal of boné and mineral research, 33(5):845-85.

Fabricio, S. C. C., Rodrigues, R. A. P. \& Costa Junior, M. L. D. (2004) Causas e conseqüências de quedas de idosos atendidos em hospital público. Revista de saúde pública, 38(1), 93-99.

Fortes, E. M, Raffaelli, M. P., Bracco, O. L., Takata, E. T. T., Reis, F. B., Santili, C. \& Castro, M. L. (2008). Elevada morbimortalidade e reduzida taxa de diagnóstico de osteoporose em idoso com fratura de fêmur proximal na cidade de São Paulo. Arquivos brasileiro de endocrinologia e metabolismo, 2(7):110614.

Frazão, P. \& Naveira, M. (2006). Prevalência de osteoporose: uma revisão crítica. Revista Brasileira de Epidemiologia, 9(2), $206-214$.

Kanis, J. A. (2007). On behalf of the World Health Organization Scientific Group. Assessment of osteoporosis at the primary health-care level. Technical report. University of Sheffield, UK: WHO Collaborating Centre.

Kanis, J. A., Cooper, C., Rizzoli, R. \& Reginster, J. Y. (2019). Scientific Advisory Board of the European Society for Clinical and Economic Aspects of Osteoporosis (ESCEO) and the Committees of Scientific.

Lanzillotti, H. S., Lanzillotti, R. S., Trotte, A. P., Dias, A. S., Bornand, B. \& Costa, E. A. M. M. (2003). Osteoporosis in postmenopausal women, dietary calcium and other risk factors. Revista de nutrição, 16(2):181-93.

Maclen, C., Alexander, A., Carter, J., Chen, S., Desai, S. B., Grossman, J., Maglione, M., Mcmahon, M., Mcmanara M., Mojica, W., Newberry, M., Ranganaht, V., Suttorp, M., Timmer, M., Tringale, C., Valentine, D. \& Zhou, A. (2007). Comparative Effectiveness of Treatments To Prevent Fractures in Men and Women With Low Bone Density or Osteoporosis. Rockville (MD): Agency for Healthcare Research and Quality (US).

Moraes, L., Silva, E., Silva, D. \& Paula, A. (2014). Gastos com o tratamento da osteoporose em idosos do Brasil (2008 - 2010): análise dos fatores associados. Revista brasileira de epidemiologia. 17(3), 719-734. 
NIH Consensus Development Panel on Osteoporosis Prevention, Diagnosis, and Therapy. Osteoporosis prevention, diagnosis, and therapy. (2001). JAMA, 285(6):785-95.

Osteoporosis 1995. Basic diagnosis and therapeutic elements for a national consensus proposal., Med J. 113(4 Suppl):1-64.

Pereira, A. S., Shitsuka, D. M., Parreira, F. J. \& Shitsuka, R. (2018). Metodologia da pesquisa cientifica. UFSM. https://repositorio.ufsm.br/bitstream/handle/1/15824/Lic_Computacao_Metodologia-Pesquisa-Cientifica.pdf?sequence=1.

Pereira, E. N. L. (2017). Análise da distribuição dos equipamentos de densitometria óssea no Brasil: importância da gestão eficiente de tecnologias em saúde. https://repositorio.unb.br/handle/10482/23334

Pereira, P. S., Pereira, F. A. \& Bohland, A. K.(2018). Prevalência de fraturas de fêmur proximal em idosos no estado de sergipe de 2000 a 2014, Universidade Federal de Sergipe.

Pinheiro, M. M., Castro, C. M. \& Szejnfeld, V. L (2006). Low femoral bone mineral density and quantitative ultrasound are risk factors for new osteoporotic fracture and total and cardiovascular mortality: a 5-year population-based study of brazilian elderly women. The journals of gerontology. Series A, Biological scienses and medical scienses, 61(2):196-203.

Pinheiro, M. M. \& Eis, S. R. (2010). Epidemiology of osteoporotic fractures in Brazil: what we have and what we need. Arquivos brasileiros de endocrinologia e metabologia. 54(2), 164-170.

Pinheiro, M. M, Ciconelli, R. M., Jacques, N., Genaro, P. S., Martini, L. A. \& Ferraz, M. B. (2010). O impacto da osteoporose no Brasil: dados regionais das fraturas em homens e mulheres adultos - The Brazilian Osteoporosis Study (BRAZOS). Revista brasileira de reumatologia. 50(2), 113-120.

Pinheiro, M. M., Neto, E. T. R., Machado, F. S., Omura, F., Yang, J. H. K., Szejnfeld, J. \& Szejnfeld, V. L. (2010). Risk factors for osteoporotic fractures and low bone density in pre and postmenopausal women. Revista de saúde pública. 44(3), 479-485.

Radominski, S. C., Bernardo, W., Paula, A.P., Albergaria, B.H., Moreira, C., Fernandes, C.E., Castro, C. H. M., Zerbini, C. A. F, Domiciano, D. S., Mendonça, L. M. C., Pompei, L. M., Bezerra, M. C., Loures M. A. R., Wender, M. C. O., Lazaretti-Castro, M., Pereira, R. M. R., Maeda, S. S., Szejnfeld, V. L. \& Borba, V. Z. C. (2017). Brazilian guidelines for the diagnosis and treatment of postmenopausal osteoporosis. Revista brasileira de reumatolologia, 57 Suppl 2:452-466.

Ralston, S. H. \& Fraser, J. (2015). Diagnosis and management of osteoporosis. Practitioner. 259(1788):15-9, 2.

Rodrigues, C. M. B., Cendoroglo, M. S., Ramos, L., Latorre M. M. M., Saraiva, G. L., Lage, A., Neto, N. C., Araujo, L. M. Q., Vieira, J. G. H., \& LazarettiCastro, M. (2005). Bone mineral density and osteoporosis among a predominantly Caucasian elderly population in the city of Sao Paulo, Brazil. Osteoporosis international, 16(11):1451-60.

Rubin, K. H., Möller, S., Holmberg, T., Bliddal, M., Søndergaard, J. \& Abrahamsen, B. (2018). A New Fracture Risk Assessment Tool (FREM) Based on Public Health Registries. Journal of bone and mineral research, 33(11):1967-1979.

Santana, J. E J., Santos, S. R. A., Santos, F. S., Nascimento, G.A., Moraes, E. J. R., Pereira, F. A. (2017). Avaliação dos fatores de risco para Osteoporose e Fratura Osteoporótica na População de Idosos da Cidade de Canhoba - Sergipe. Archives of endocrinology and metabolism, 61(66)

Sernbo, I. \& Johnell, O. (1993). Consequences of a hip fracture: a prospective study over 1 year. Osteoporosis international, 3(3):148-53.

Siris, E. S., Miller, P. D., Barrett-Connor, E., Faulkner, K. G., Wehren, L. E., Abbott, T. A., Berger, M. L., Santora, A. C. \& Sherwood, L.M. (2001). Identification and fracture outcomes of undiagnosed low bone mineral density in postmenopausal women: results from the National Osteoporosis Risk Assessment. JAMA, 12;286(22):2815-22.

Szejnfeld, V. L., Jennings, F., Castro, C. H. M., Pinheiro, M. M. \& Lopes, A. C. (2007). Conhecimento dos médicos clínicos do Brasil sobre as estratégias de prevenção e tratamento da osteoporose. Revista brasileira de reumatologia, 47(4):251-7.

Takkouche, B., Montes-Martinez, A., Gill, S. S. \& Etminan, M. (2007). Psychotropic medications and the risk of fracture: a meta-analysis. Drug safety, 30(2):171-84.

Turner, D. A., Khioe, R. F. S., Shepstone, L., Lenaghan, E., Cooper, C., Gittoes, N., Harvey, N. C., Holland, R., Howe, A., McCloskey, E., O'Neill, T. W., Torgerson, D. \& Fordham, R.J. (2018). The Cost-

Vestergaard, P., Rejnmark, L. \& Mosekilde, L. (2005). Osteoporosis is markedly underdiagnosed: a nationwide study from Denmark. Osteoporosis international, 16(2):134-41. 